López-Aguilar, D., Álvarez-Pérez, P.R. y Ravelo-González, Y. (2022). Capacidad de adaptabilidad e intención de abandono académico en estudiantes universitarios. Revista de Investigación Educativa, 40(1), 237-255.

DOI: http://dx.doi.org/10.6018/rie.463811

\title{
Capacidad de adaptabilidad e intención de abandono académico en estudiantes universitarios
}

\author{
Adaptability skills and the intention to drop out in university \\ students
}

\author{
David López-Aguilar, Pedro Ricardo Álvarez-Pérez' y Yennifer Ravelo-González \\ Universidad de la Laguna (España)
}

\begin{abstract}
Resumen
La finalidad de esta investigación fue analizar la relación entre dos factores de especial relevancia para los procesos de enseñanza-aprendizaje en la educación superior durante el confinamiento por la COVID-19: la capacidad de adaptabilidad y la intención de abandono. Los efectos de la pandemia se han dejado sentir en los contextos educativos y en el caso de la enseñanza universitaria, ha supuesto el cambio de un modelo de enseñanza presencial a otro totalmente virtual. Por este motivo, el objetivo de la investigación se orientó a comprobar si los estudiantes con menores competencias de adaptabilidad para hacer frente a las nuevas condiciones educativas impuestas por la pandemia, tenían mayores intenciones de abandonar la formación. La recogida de datos se llevó a cabo mediante un cuestionario on-line, respondido por una muestra de 1519 estudiantes de primero y segundo curso de todas las titulaciones de grado de la Universidad de La Laguna (ULL). Los resultados confirmaron que un porcentaje considerable de alumnado se planteó abandonar la formación universitaria que había comenzado, especialmente los matriculados en titulaciones del ámbito de las Ciencias Sociales y Jurídicas. Los estudiantes con mejores calificaciones son los que mostraron menor intención de abandono. Por otro lado, cuanto mayor eran las puntuaciones obtenidas en las dimensiones de adaptabilidad (preocupación, control, curiosidad y confianza), menor era la intención de abandono. Del estudio se derivan implicaciones importantes para la práctica,
\end{abstract}

1 Correspondencia: Pedro Ricardo Álvarez-Pérez, palvarez@ull.edu.es, Universidad de La Laguna (España). 
formando al alumnado en competencias de adaptabilidad para hacer frente a los cambios $y$ diferentes situaciones relacionadas con su proceso formativo.

Palabras clave: estudiantes universitarios; abandono de los estudios; adaptabilidad; motivación para los estudios; prácticas de enseñanza.

\begin{abstract}
The purpose of this research was to analyse the relationship between two factors of special relevance for the teaching-learning processes in higher education during the confinement caused by COVID-19: the capacity to adapt and the intention to drop out. The effects of the pandemic have been felt in educational contexts and, in the case of university education, it has led to the change from a face-to-face teaching model to a totally virtual one. For this reason, the objective of the research was aimed at verifying whether students with lower adaptability skills to cope with the new educational conditions imposed by the pandemic had greater intentions to abandon training. The data collection was carried out by means of an online questionnaire, answered by a sample of 1519 first-and second-year students from all undergraduate degrees at the University of La Laguna. The results confirmed that a considerable percentage of students considered abandoning the university education they had begun, especially those enrolled in degrees in the field of Social and Legal Sciences. The students with the best grades are those who showed the least intention to drop out. On the other hand, the higher the scores obtained in the adaptability dimensions (concern, control, curiosity and confidence), the lower the intention to quit. Important implications for practice are derived from the study, such as training students in adaptability skills to cope with changes and different situations related to their training process.
\end{abstract}

Keywords: university students; drop out; adaptability; learning motivation; teaching methods.

\title{
Introducción
}

No cabe duda de que una de las características que define el modelo de sociedad actual es la transformación y el cambio permanente que tiene lugar en todos los ámbitos que la conforman. Y uno de los ámbitos sometidos a continuos cambios es el educativo, lo que supone para el alumnado que accede a la educación superior un desafío, en muchos casos difícil de superar. Iniciar estudios universitarios es avanzar un paso más en la trayectoria formativa y supone integrarse en una nueva cultura académica, asimilar nuevos hábitos, nuevas formas de aprender, nuevas metodologías, nuevas dinámicas, nuevos espacios de socialización, etc. (Romero y Figuera, 2016). El desarrollo vocacional se organiza a partir de una red de decisiones, identidades y roles que cada persona asume y para ello se necesita una buena capacidad de adaptación y anticipación estratégica desde la que se pueda hacer frente a los imprevisibles y rápidos cambios (Ambiel et al., 2016; García-Segura et al., 2017).

$\mathrm{Y}$ al igual que la adaptabilidad profesional es una competencia fundamental para que los trabajadores aborden de manera satisfactoria sus trayectorias de carrera, también los estudiantes deben dominar la capacidad de adaptarse a las diversas realidades de la vida universitaria, afrontando con responsabilidad sus decisiones, 
explorando oportunidades, adquiriendo nuevas habilidades y teniendo confianza en sus posibilidades para desarrollar su proyecto académico-profesional de carrera (Santana et al., 2019). Este es un reto clave, porque muchos estudiantes llegan a la universidad con su particular conjunto de características, con una visión no siempre real de lo que supone ser estudiante universitario $y$, a veces, con poca claridad sobre las metas y el procedimiento para conseguirlas (Martínez-Clares et al., 2014). Solo cuando comienzan los estudios se dan cuenta de que no poseen las capacidades adecuadas para abordar las tareas académicas o que la titulación elegida no les satisface, por lo que la adaptación es muy compleja y muchos terminan abandonando (Bernardo et al., 2020; Figuera et al., 2015). Por diversos motivos, muchos estudiantes no son capaces de superar el desafío que implica cursar estudios universitarios y se sienten extraños, desmotivados, poco reconocidos, excluidos y no terminan su proceso de adaptación (Freixa et al., 2018; Gonzalo y García, 2016). Rausch y Hamilton (2006) identificaron como factores asociados al abandono, la percepción de falta de integración, las dificultades para socializarse y adaptarse y la sensación de aislamiento social. Cuando el estudiante percibe esta sensación de aislamiento, se plantea la desvinculación académica, que se vive como una liberación de la presión asociada al proceso formativo (Bethencourt et al., 2008; Tuero et al., 2018).

El abandono de los estudios es uno de los problemas más graves a los que se enfrentan las instituciones universitarias (Álvarez y Cabrera, 2020; García-Aretio, 2019; González y Pedraza, 2017). La tasa de abandono constituye un claro indicador de calidad, contemplado en diferentes modelos de evaluación de la educación superior (Cabrera et al., 2006), por lo que se insiste en la necesidad de encontrar soluciones a este grave problema, que afecta tanto al alumnado, como a la familia, a la institución y a la sociedad en general. Los estudios que se vienen realizando en torno a este fenómeno, señalan que se trata de un problema multicausal. El abandono se relaciona con variables como: sentido de pertenencia (Martínez et al., 2014), expectativas de realización (Marín et al., 2000), gestión del aprendizaje (Barefoot, 2004), creencias de autoeficacia (Betz y Hackett, 1981), motivación hacia los estudios (Landry, 2003), compromiso académico (Zepke y Leach, 2010), asistencia a clase (Álvarez y López, 2011), capacidad de adaptación al contexto universitario (Esteban et al., 2016), etc.

De entre los distintos factores que se relacionan con el abandono académico, queremos centrarnos en las competencias de adaptabilidad (Nilforooshan y Salimi, 2016; Perera y McIlveen, 2017). Cabrera et al. (2006) identificaron como causas de la deserción el bajo interés vocacional, la escasa motivación y el bajo dominio de habilidades para adaptarse a los cambios. Para Savickas y Porfeli (2012), la adaptación está motivada por el objetivo de encontrar el equilibrio entre las necesidades internas y las oportunidades externas. La adaptabilidad exige el dominio de determinadas competencias para hacer frente a las transiciones, a los requerimientos académicos, al enfrentamiento de nuevos retos, etc. La adaptación es el resultado de las conductas que las personas manifiestan frente a los cambios. Las personas con competencias de adaptabilidad se caracterizan por presentar preocupación por el futuro, tener control sobre las situaciones, mostrar curiosidad por explorar posibles identidades y nuevas oportunidades y por tener confianza al iniciar proyectos relacionados con sus aspiraciones personales y profesionales (Savickas, 2005). Tendrán mejores resultados de 
adaptación, aquellos que son capaces de tener conductas para enfrentar condiciones y situaciones cambiantes (Savickas y Porfeli, 2012).

En el campo de la educación, los estudiantes con capacidad de adaptabilidad confían en sus posibilidades y manejan con éxito los ajustes necesarios para afrontar los cambios de cursos y etapas educativas (Mamani, 2017). Además, como confirman Wilkins-Yel et al. (2018), la adaptabilidad de carrera se relaciona de forma positiva con la permanencia en los estudios, la afrontación de los imprevistos y la satisfacción académica. Los efectos recientes de la pandemia por la COVID-19 han producido cambios sustanciales en los procesos educativos, que han puesto de manifiesto la importancia de saber adaptarse a los mismos. El tránsito apresurado de una enseñanza presencial a una virtual, ha obligado al alumnado a un proceso de adaptación rápida. A muchos estudiantes esta situación les ha generado tensión, estrés, incertidumbre o dudas respecto a si serían capaces de cumplir con las exigencias del aprendizaje en este nuevo escenario formativo (Pérez-López et al., 2021; Salavera y Usán, 2017). Los que no están preparados y no poseen buenas competencias de adaptabilidad, ante situaciones de esta naturaleza, pueden verse abocados a la deserción académica (Himmel, 2018).

\section{Método}

\section{Objetivos e hipótesis}

El objetivo general de la investigación fue analizar si los estudiantes con baja capacidad de adaptabilidad durante el confinamiento tenían mayor intención de abandono de los estudios. La hipótesis de trabajo se basó en que, aquellos estudiantes con baja capacidad de adaptación a los procesos de enseñanza y aprendizaje telemáticos derivados de la pandemia tenían mayor intención de abandonar los estudios $\left(\mathrm{H}_{1}\right)$, mientras que el alumnado con estrategias adecuadas para la adaptación, tendrían éxito y permanecerían en su formación $\left(\mathrm{H}_{2}\right)$.

\section{Participantes}

Formaron parte de la población aquellos estudiantes que realizaban el primer o segundo curso de los grados oficiales de la ULL. Según datos del Gabinete de Análisis y Planificación (GAP) de la ULL, durante el curso 2019/2020 había matriculados un total de 8753 estudiantes en estos cursos. Para asegurar un nivel de confianza del 95\% y un margen de error de \pm 5 , se debía contar con una muestra total de 369 estudiantes. Mediante un procedimiento de muestreo no aleatorio accidental, en este estudio participaron 1519 estudiantes, lo que permitió alcanzar una confianza del 99.0\% y situar el rango de error en \pm 3.00 . A partir de los criterios de selección establecidos, las características básicas de la muestra se presentan en la tabla 1. 
Tabla 1

Características de la muestra

\begin{tabular}{ll}
\hline Género & Hombres: $30.0 \%(\mathrm{n}=456)$ \\
& Mujeres:70.0\%(n=1063) \\
\hline Edad & $=20.34$ \\
& sd=4.02 \\
\hline Curso & Primero: $54.4 \%(\mathrm{n}=826)$ \\
& Segundo: $45.6 \%(\mathrm{n}=693)$ \\
\hline Rama de conocimiento & Artes y Humanidades: $18.4 \%(\mathrm{n}=280)$ \\
& Ciencias: $12.4 \%(\mathrm{n}=188)$ \\
& Ciencias de la Salud:13.6\%(n=206) \\
& Ciencias Sociales y Jurídicas: $41.1 \%(\mathrm{n}=624)$ \\
& Ingeniería y Arquitectura: $14.5 \%(\mathrm{n}=221)$ \\
\hline
\end{tabular}

\section{Instrumento de recogida de datos}

El "Cuestionario de intención de abandono académico en la enseñanza universitaria" se construyó ad hoc incluyendo, entre otras preguntas, la Career Adapt-Abilities Scale de Savickas y Porfeli (2012). Esta escala tipo Likert mide la capacidad de adaptabilidad a través de 24 ítems distribuidos en cuatro factores (tabla 2): preocupación, control, curiosidad y confianza. Según Savickas y Porfeli (2012), los niveles de fiabilidad medidos a través de la prueba alfa de Cronbach superaron los valores críticos establecidos por la literatura (Oviedo y Campo-Arias, 2005). Las puntuaciones $\alpha$ obtenidas fueron preocupación:.90, control:.89, curiosidad:.89 y confianza:.91. En cuanto a la estructura factorial de la escala de adaptabilidad, los análisis factoriales exploratorios y confirmatorios presentados por Savickas y Porfeli (2012), muestran que los valores (CFI, RMSEA, SRMR) cumplieron con los requisitos propuestos por la literatura.

Tabla 2

Dimensiones e ítems de la escala de adaptabilidad

\begin{tabular}{lll}
\hline Dimensión & \multicolumn{1}{c}{ Ítems } & Cod. \\
\hline \multirow{2}{*}{ Preocupación } & Piensas sobre cómo será tu futuro & Pre1 \\
\cline { 2 - 3 } & $\begin{array}{ll}\text { Eres consciente que las decisiones que tomes hoy condicionarán tu } \\
\text { futuro }\end{array}$ & Pre2 \\
\cline { 2 - 3 } & Te estás preparando para el futuro profesional & Pre3 \\
\cline { 2 - 3 } & Eres consciente de las decisiones vocacionales que debes tomar & Pre4 \\
\cline { 2 - 3 } & Planeas cómo conseguir tus objetivos académico-profesionales & Pre5 \\
\cline { 2 - 3 } & Te mantienes optimista ante tu futuro académico-profesional & Pre6 \\
\hline
\end{tabular}




\begin{tabular}{|c|c|c|}
\hline Dimensión & Ítems & Cod. \\
\hline \multirow[t]{6}{*}{ Control } & Estás preocupado por tu desarrollo académico-profesional & Con1 \\
\hline & Tomas decisiones por ti mismo & Con2 \\
\hline & Asumes la responsabilidad de tus actos & Con3 \\
\hline & Te mantienes fiel a tus principios & Con4 \\
\hline & Confías en ti mismo & Con5 \\
\hline & $\begin{array}{l}\text { Haces lo que está bien para tu trayectoria y proyecto académico-pro- } \\
\text { fesional }\end{array}$ & Con6 \\
\hline \multirow[t]{6}{*}{ Curiosidad } & Exploras tu entorno & Cu1 \\
\hline & Buscas oportunidades de crecimiento como persona & $\mathrm{Cu} 2$ \\
\hline & Investigas las opciones antes de tomar una decisión & Cu3 \\
\hline & $\begin{array}{l}\text { Observas las diferentes vías y alternativas para lograr tus objetivos } \\
\text { académicos y profesionales }\end{array}$ & $\mathrm{Cu} 4$ \\
\hline & Intentas buscar respuesta a tus inquietudes académicas & $\mathrm{Cu} 5$ \\
\hline & La curiosidad te mueve a buscar nuevas oportunidades & Cu6 \\
\hline \multirow[t]{6}{*}{ Confianza } & Realizas eficientemente tus tareas académicas & Conf1 \\
\hline & Eres cuidadoso para hacer las cosas de clase bien & Conf2 \\
\hline & Aprendes nuevas habilidades que te sirven para tu proceso formativo & Conf3 \\
\hline & Trabajas de acuerdo con tus posibilidades & Conf4 \\
\hline & Superas los obstáculos que encuentras en tu proceso formativo & Conf5 \\
\hline & Resuelves los problemas relacionados con tu proceso formativo & Conf6 \\
\hline
\end{tabular}

Además de esta escala, se incluyeron otras preguntas de carácter sociodemográfico y académico (género, edad, curso, titulación, calificaciones obtenidas en la universidad, nivel de motivación, intención de abandono y motivos de intención de abandono) para describir los principales rasgos de la muestra participante y ser usadas en los análisis de contraste. Las medidas empleadas en las preguntas del cuestionario se presentan en la tabla 3.

Tabla 3

Medidas empleadas

\begin{tabular}{ll}
\hline Ítem & Medida empleada \\
\hline Género & Dicotómica \\
\hline Edad & Abierta \\
\hline Curso & Elección múltiple \\
\hline Titulación & Elección múltiple \\
\hline Calificaciones & Elección múltiple \\
\hline Motivación & Escala Likert (1-7) \\
\hline Intención de abandono & Dicotómica \\
\hline Motivos de intención de abandono & Abierta \\
\hline Escala de adaptabilidad & Escala Likert (1-7) \\
\hline
\end{tabular}


Como paso previo a la administración definitiva del instrumento, y dado que la escala incluida en el cuestionario iba a ser adaptada y utilizada en estudiantes universitarios, se tradujeron los ítems de la Career Adapt-Abilites Scale la cual fue sometida, junto al resto de preguntas del cuestionario a las propuestas que McMillan y Schumacher (2005) hacen para el diseño de técnicas de recogida de datos:

- En primer lugar, se llevó a cabo una prueba de expertos en la que participaron 3 profesores universitarios especializados en el objeto de esta investigación, que valoraron, ítem a ítem, la comprensión, pertinencia e idoneidad.

- Por otro lado, se realizó una prueba de forma, en la que 2 expertos del campo de la metodología de la investigación educativa valoraron, además de la comprensión, pertinencia e idoneidad, cuestiones referidas a la adecuación de las preguntas a los objetivos del estudio, si los ítems estaban debidamente construidos para el tipo de análisis que se pretendían realizar, etc.

Para llevar a cabo estas dos pruebas, se construyó un instrumento en el que los evaluadores participantes valoraron la pertinencia, claridad e idoneidad (en una escala tipo Likert de 1 a 7 (1 valoración más baja; 7 valoración más alta)) de cada uno de los ítems propuestos. Asimismo, las distintas preguntas incluidas en el cuestionario fueron valoradas cualitativamente a través de un apartado de observaciones.

Además, se realizó una prueba piloto con una muestra de 21 estudiantes que reunían los requisitos establecidos para participar en este estudio. En esta prueba se analizaron los tiempos de respuesta del instrumento, la claridad y comprensión de cada uno de los ítems, etc. Durante el desarrollo de esta prueba, se comprobó que los ítems de la escala de adaptabilidad que habían sido traducidos eran comprendidos correctamente por los participantes manteniendo el sentido y finalidad original.

La realización de estas pruebas permitió incorporar modificaciones en el instrumento definitivo. Los coeficientes de fiabilidad derivados de la aplicación de este instrumento de medida en este estudio se presentan en los resultados.

\section{Procedimiento de recogida de datos}

Finalizada la construcción del instrumento, se estableció el procedimiento de recogida de datos que tuvo lugar durante los meses de febrero a mayo de 2020. A partir de las medidas implantadas en la ULL para desarrollar los procesos de enseñanza y aprendizaje de manera telemática como consecuencia de la COVID-19, se llevó a cabo la recogida de datos a través de un sistema online mediante la herramienta Google Formularios que incluía las preguntas del cuestionario definido. Para el envío de esta prueba se contactó a través de correo electrónico con la totalidad del profesorado de la ULL que impartía su docencia en los cursos de primero y segundo de grado. En este mensaje se explicaba al profesorado las finalidades de este estudio y se solicitaba su colaboración para que aplicara el cuestionario en sus clases online. Asimismo, y teniendo en cuenta procedimientos de ética, se adjuntó al profesorado un texto que compartió con el alumnado en el momento de administrar la prueba en el que se informó a los estudiantes sobre la finalidad de la investigación, se solicitó su participación voluntaria y se garantizó el anonimato de sus respuestas. 


\section{Análisis de datos}

Recogida la información, se descargó la base de datos generada automáticamente en la aplicación Google Formularios. Este fichero fue tratado con el software R-Studio (versión 1.3.1093) para el sistema operativo Microsoft Windows 10. Este programa permitió realizar análisis de distinta naturaleza: preparación de la base de datos, estudio de la distribución de los datos y cálculo de la fiabilidad de la escala de medida. Además, se realizaron cálculos de tendencia central, frecuencias, análisis de contraste con tablas de contingencia y pruebas no paramétricas. También se llevó a cabo un análisis de contenido de la pregunta abierta incluida en el cuestionario codificada con las siglas $p N$ ( $\mathrm{p}=$ participante; $\mathrm{N}=$ número de sujeto del estudio).

Para el cálculo del tamaño del efecto de las diferencias encontradas en la prueba U de Mann-Whitney, se utilizó el programa Microsoft Excel (versión Office 365) para el entorno Microsoft Windows 10. Este cálculo se realizó con la prueba de probabilidad de superioridad $\left(\mathrm{PS}_{\text {est }}\right.$ ) cuya expresión matemática es (Erceg-Hurn y Mirosevich, 2008):

$$
P S_{e s t}=\frac{U}{m \cdot n}
$$

Para los análisis realizados el valor $\alpha$ se fijó en .05.

\section{Resultados}

\section{Preparación de la base de datos y análisis preliminares}

Inicialmente se preparó la base de datos a emplear y se llevaron a cabo cálculos preliminares para tomar decisiones sobre los análisis de contraste a ejecutar. El primer paso consistió en comprobar que la información imputada en los ítems estuviera dentro del rango esperado. Finalizada esta revisión, se valoró la posible existencia de información perdida. En este caso, la base de datos se generó de manera automática a partir de la herramienta de Google Formularios, por lo que no se identificó.

Otro de los aspectos que se analizó fue la presencia de casos extremos mediante la distancia de Mahalanobis. El cálculo de esta distancia arroja una puntuación crítica a partir de la cual los sujetos son considerados como extremos (Muñoz y Amón, 2013). La distancia de Mahalanobis se situó en un valor de 76.777, lo que sugirió la eliminación de 98 participantes. De este modo, la muestra definitiva quedó configurada por un total de 1421 estudiantes universitarios.

Con la finalidad de confirmar que las preguntas del cuestionario discriminaron correctamente y no existió redundancia, se llevó a cabo un análisis de la multicolineidad de los ítems a través de una correlación bivariada, que arrojó valores, para todos los casos $r \leq .85$, lo que según Holgado et al. (2019) se situaron por debajo de los valores críticos establecidos.

Para la selección de las pruebas estadísticas a realizar (paramétricas o no paramétricas) se llevó a cabo un estudio de la normalidad a través de la prueba KolmogorovSmirnov (KS). Los resultados de este examen estadístico, para la totalidad de los casos, 
arrojaron valores $\mathrm{p}<.000$. Esto indició que los datos no se ajustaron a un patrón de distribución normal (George y Mallery,2011).

Finalmente, se realizaron cálculos de fiabilidad de la escala de medida. Dado que la escala cumplió con los supuestos de tau-equivalencia, unidimensionalidad y continuidad de medida (Raykov y Marcoulides, 2017) se llevó a cabo la prueba alfa de Cronbach. Además, y siguiendo los planteamientos de Viladrich et al. (2017), se realizó el cálculo del coeficiente Omega de MacDonald, por tener una mayor eficiencia y robustez en estudios relacionados con el ámbito de las ciencias sociales. Los valores de fiabilidad (tabla 5) superaron las puntuaciones críticas propuestas por Oviedo y Campo-Arias (2005).

Tabla 5

Análisis de fiabilidad

\begin{tabular}{lcc}
\hline Dimensión & Alfa de Cronbach $(\boldsymbol{\alpha})$ & Omega de MacDonald $(\omega)$ \\
\hline Preocupación & .89 & .92 \\
\hline Control & .88 & .94 \\
\hline Curiosidad & .93 & .96 \\
\hline Confianza & .94 & .96 \\
\hline Total & .97 & .97 \\
\hline
\end{tabular}

\section{El alumnado con intención de abandono de los estudios}

El 39.9\% de los participantes manifestó tener intención de abandonar los estudios universitarios que estaba cursando. En las respuestas ofrecidas en las preguntas abiertas se comprobó que algunos de los principales motivos que llevaron al alumnado a pensar en abandonar su formación estaban referidos principalmente a situaciones económicas complejas en el seno de la familia, a presiones externas (dificultades académicas, apoyos de la familia y amistades, etc.) y desmotivación por los estudios. Así lo reflejaron los relatos ofrecidos por los estudiantes al indicar que "no me está yendo nada bien en cuanto a resultados académicos se refiere" (p879), "sin beca no puedo seguir estudiando"(p412), "recibo poco apoyo por parte de la familia y amigos [...] y por la desmotivación que te transmiten los profesores"(p1023), "no me adapto bien, tengo dificultades, estoy desmotivado"(p268) "supone mucha presión y son estudios difíciles que requieren una alta carga de trabajo"(p693).

Los análisis de contraste revelaron que los niveles de intención de abandono académico eran mayores entre el alumnado que cursaba titulaciones de la rama de Ciencias Sociales y Jurídicas $\left(X^{2}=12.519 ; \mathrm{p}=.014\right)$ con un $37.4 \%$, seguido de Artes y Humanidades (21.2\%), Ingeniería y Arquitectura (16.9\%) y Ciencias (12.7\%). El conjunto de estudiantes con menor intención de abandono fue el de Ciencias de la Salud (11.8\%).

Asimismo, el alumnado con calificaciones promedio de sobresaliente y matrícula de honor en las asignaturas que cursó en la enseñanza universitaria fueron los que tuvieron un menor índice de abandono $\left(\mathrm{X}^{2}=113.252 ; \mathrm{p}<.000\right)$ con un .5\% y $2.1 \%$ respecti- 
vamente. Fue el conjunto de estudiantes con calificaciones de aprobado los que mayor predisposición tenían hacia la deserción de los estudios (39.5\%).

En cuanto a la motivación del alumnado, los estudiantes con riesgo de abandono presentaron un menor nivel de interés hacia la formación universitaria que cursaban $\left(\mathrm{U}=110623.5 ; \mathrm{R}=479.10 ; \mathrm{p}<.000 ; \mathrm{PS}_{\text {est }}=.228\right)$.

\section{Adaptabilidad e intención de abandono académico del alumnado universitario}

En términos generales, los niveles de adaptabilidad del alumnado durante el tiempo de confinamiento fueron moderados (rango medio=4). En un análisis más pormenorizado, se comprobó que estas valoraciones también se reflejaron en las distintas dimensiones que configuraban la escala de adaptabilidad. Así, en la dimensión preocupación el alumnado obtuvo un rango medio de 4 . Ahondando sobre los ítems de esta dimensión (tabla 5), se comprobó que el alumnado era consciente de la importancia de las decisiones que tomaba hoy para su futuro profesional (rango medio $=4$ ), pensaba en cómo sería su devenir (rango medio=4) y sabía que se estaba preparando para su futuro profesional (rango medio $=4$ ).

Tabla 6

Dimensión preocupación

\begin{tabular}{lc}
\hline Ítems & Rango medio \\
\hline Preocupación general & 4 \\
\hline Pre1 & 4 \\
\hline Pre2 & 4 \\
\hline Pre3 & 4 \\
\hline Pre4 & 4 \\
\hline Pre5 & 4 \\
\hline Pre6 & 4 \\
\hline
\end{tabular}

El análisis de contraste realizado en esta primera dimensión (tabla 7) demostró que el alumnado que tenía un nivel de adaptabilidad más bajo, era también el que mostró una mayor intención de abandono de los estudios ( $\mathrm{U}=181049.000 ; \mathrm{R}=603.31 ; \mathrm{p}<.000$; PSest=.373). De hecho, el conjunto de estudiantes que manifestó su intención de abandonar su formación fue el que demostró tener una menor preparación para su futuro profesional $\left(\mathrm{U}=209119.000 ; \mathrm{R}=652.82 ; \mathrm{p}<.000 ; \mathrm{PS}_{\mathrm{est}}=.431\right)$, el que menos consciente era de las decisiones vocacionales que debía tomar $\left(\mathrm{U}=214370.000 ; \mathrm{R}=662.08 ; \mathrm{p}<.000 ; \mathrm{PS}_{\text {est }}=.442\right)$, el que valoró con puntuaciones más bajas la necesidad de planificar el logro de sus objetivos académico-profesionales ( $\left.\mathrm{U}=204997.500 ; \mathrm{R}=645.55 ; \mathrm{p}<.000 ; \mathrm{PS}_{\text {est }}=.423\right)$ y el que se mantuvo menos optimista ante su futuro académico y profesional ( $U=151399.500$; $\mathrm{R}=551.02 ; \mathrm{p}<.000 ; \mathrm{PS}_{\text {est }}=.312$ ). 
Tabla 7

Análisis de contraste preocupación

\begin{tabular}{|c|c|c|c|c|c|}
\hline Ítems & Intención de abandono & Rango promedio & $\mathrm{U}$ & $p$ & PSest \\
\hline \multirow{2}{*}{ Pre1 } & No & 725.83 & \multirow{2}{*}{229446.500} & \multirow{2}{*}{.054} & \\
\hline & Sí & 688.67 & & & \\
\hline \multirow{2}{*}{ Pre2 } & No & 724.89 & \multirow{2}{*}{230248.500} & \multirow{2}{*}{.72} & \\
\hline & Sí & 690.08 & & & \\
\hline \multirow{2}{*}{ Pre3 } & No & 749.63 & \multirow{2}{*}{209119.000} & \multirow{2}{*}{$<.000$} & \multirow{2}{*}{.431} \\
\hline & Sí & 652.82 & & & \\
\hline \multirow{2}{*}{ Pre4 } & No & 743.48 & \multirow{2}{*}{214370.000} & \multirow{2}{*}{$<.000$} & \multirow{2}{*}{.442} \\
\hline & Sí & 662.08 & & & \\
\hline \multirow{2}{*}{ Pre5 } & No & 754.46 & \multirow{2}{*}{204997.500} & \multirow{2}{*}{$<.000$} & \multirow{2}{*}{.423} \\
\hline & Sí & 645.55 & & & \\
\hline \multirow{2}{*}{ Pre6 } & No & 817.22 & \multirow{2}{*}{151399.500} & \multirow{2}{*}{$<.000$} & \multirow{2}{*}{.312} \\
\hline & Sí & 551.02 & & & \\
\hline
\end{tabular}

En cuanto al control, los datos mantuvieron una tendencia promedio similar al resto (rango medio $=4$ ). De manera específica (tabla 8), el alumnado destacó sobre todo por la capacidad para asumir la responsabilidad de sus actos (rango medio=4), el mantenerse fiel a sus propios principios (rango medio $=4$ ) y el tener la habilidad de tomar decisiones por sí mismos (rango medio=4).

Tabla 8

Dimensión control

\begin{tabular}{lc}
\hline Ítems & Rango medio \\
\hline Control_general & 4 \\
\hline Con1 & 4 \\
\hline Con2 & 4 \\
\hline Con3 & 4 \\
\hline Con4 & 4 \\
\hline Con5 & 4 \\
\hline Con6 & 4 \\
\hline
\end{tabular}

En esta dimensión (tabla 9), se encontró que el alumnado que manifestó tener intención de abandonar los estudios fue el que obtuvo puntuaciones más bajas ( $\mathrm{U}=19$ $\left.2736.500 ; \mathrm{R}=623.92 ; \mathrm{p}<.000 ; \mathrm{PS}_{\text {est }}=.398\right)$. Concretamente, mostraron menos habilidad para tomar decisiones por sí mismo (U=214545.500;R=662.39; $\mathrm{p}<.000 ; \mathrm{PSest}=.443)$, asumir la responsabilidad de sus actos $(\mathrm{U}=223933.000 ; \mathrm{R}=678.94 ; \mathrm{p}=.006 ; \mathrm{PSest}=.462)$, mantenerse fiel a sus principios $(\mathrm{U}=219496.000 ; \mathrm{R}=671.12 ; \mathrm{p}=.001 ; \mathrm{PSest}=.453)$, confiar en sí mismos $(\mathrm{U}=176308.000 ; \mathrm{R}=594.95 ; \mathrm{p}<.000 ; \mathrm{PSest}=.364)$ y hacer lo que está bien para su desarrollo y trayectoria académico-profesional ( $\mathrm{U}=189282.500 ; \mathrm{R}=617.83 ; \mathrm{p}<.000$; PSest=.390). 
Tabla 9

Análisis de contraste control

\begin{tabular}{|c|c|c|c|c|c|}
\hline Ítems & Intención de abandono & Rango promedio & $\mathrm{U}$ & $p$ & PSest \\
\hline \multirow{2}{*}{ Con1 } & No & 695.90 & \multirow{2}{*}{229214.500} & \multirow{2}{*}{.070} & \\
\hline & Sí & 733.74 & & & \\
\hline \multirow{2}{*}{ Con2 } & No & 743.28 & \multirow{2}{*}{214545.500} & \multirow{2}{*}{$<.000$} & \multirow{2}{*}{.443} \\
\hline & Sí & 662.39 & & & \\
\hline \multirow{2}{*}{ Con3 } & No & 732.28 & \multirow{2}{*}{223933.000} & \multirow{2}{*}{.006} & \multirow{2}{*}{.462} \\
\hline & Sí & 678.94 & & & \\
\hline \multirow{2}{*}{ Con 4} & No & 737.48 & \multirow{2}{*}{219496.000} & \multirow{2}{*}{.001} & \multirow{2}{*}{.453} \\
\hline & Sí & 671.12 & & & \\
\hline \multirow{2}{*}{ Con5 } & No & 788.05 & \multirow{2}{*}{176308.000} & \multirow{2}{*}{$<.000$} & \multirow{2}{*}{.364} \\
\hline & Sí & 594.95 & & & \\
\hline \multirow{2}{*}{ Con6 } & No & 772.86 & \multirow{2}{*}{189282.500} & \multirow{2}{*}{$<.000$} & \multirow{2}{*}{.390} \\
\hline & Sí & 617.83 & & & \\
\hline
\end{tabular}

Respecto a la curiosidad, el rango medio se situó en 4 (rango medio=4). En concreto (tabla 9), el alumnado buscó respuesta a sus inquietudes formativas (rango medio=4), observó las diferentes alternativas para hacer las cosas y lograr sus objetivos (rango medio=4) e investigó distintas opciones antes de tomar una decisión (rango medio=4).

Tabla 10

Dimensión curiosidad

\begin{tabular}{lc}
\hline Ítems & Rango medio \\
\hline Curiosidad general & 4 \\
\hline $\mathrm{Cu} 1$ & 4 \\
\hline $\mathrm{Cu} 2$ & 4 \\
\hline $\mathrm{Cu} 3$ & 4 \\
\hline $\mathrm{Cu} 4$ & 4 \\
\hline $\mathrm{Cu} 5$ & 4 \\
\hline $\mathrm{Cu} 6$ & 4 \\
\hline
\end{tabular}

La prueba U de Mann-Whitney sugirió que existían diferencias en esta dimensión (tabla 11) entre el alumnado que tenía intención de abandonar los estudios y aquellos que pretendían permanecer y finalizar su formación académica. Así, el conjunto de estudiantes con intención de desertar obtuvo puntuaciones más bajas en esta dimensión ( $\left.\mathrm{U}=129809.000 ; \mathrm{R}=624.05 ; \mathrm{p}<.000 ; \mathrm{PS}_{\text {est }}=268\right)$. Además, el alumnado que mostró su idea de abandonar los estudios fue el que menor puntuación obtuvo en la totalidad de ítems integrados en esta dimensión. De esta manera, eran los estudiantes que menos exploraban su entorno $\left(\mathrm{U}=193472.000 ; \mathrm{R}=625.22 ; \mathrm{p}<.000 ; \mathrm{PS}{ }_{\text {est }}=.399\right)$, que concedieron menor importancia a la búsqueda de oportunidades de crecimiento como 
persona $\left(\mathrm{U}=198486.000 ; \mathrm{R}=634.06 ; \mathrm{p}<.000 ; \mathrm{PS}_{\text {est }}=.409\right)$, que investigaban poco las opciones antes de tomar decisiones $(\mathrm{U}=200660.500 ; \mathrm{R}=637.90 ; \mathrm{p}<.000 ; \mathrm{PS}$ est $=.414$ ), que analizaban en menor medida las distintas alternativas para lograr sus objetivos académicos y profesionales $\left(\mathrm{U}=212475.000 ; \mathrm{R}=650.69 ; \mathrm{p}<.000 ; \mathrm{PS}_{\text {est }}=.438\right)$, que no mostraban una preocupación alta por buscar respuesta a sus inquietudes académicas ( $U=212475.000$; $\left.\mathrm{R}=658.74 ; \mathrm{p}<.000 ; \mathrm{PS}_{\text {est }}=.438\right)$ y que mostraban menos curiosidad por buscar nuevas oportunidades $\left(\mathrm{U}=210475.000 ; \mathrm{R}=655.69 ; \mathrm{p}<.000 ; \mathrm{PS}_{\mathrm{est}}=.434\right)$.

Tabla 11

Análisis de contraste curiosidad

\begin{tabular}{|c|c|c|c|c|c|}
\hline Ítems & Intención de abandono & Rango promedio & $\mathrm{U}$ & $p$ & PSest \\
\hline \multirow{2}{*}{ Cu1 } & No & 767.95 & \multirow{2}{*}{193472.000} & \multirow{2}{*}{$<.000$} & \multirow{2}{*}{.399} \\
\hline & Sí & 625.22 & & & \\
\hline \multirow{2}{*}{$\mathrm{Cu} 2$} & No & 762.08 & \multirow{2}{*}{198486.000} & \multirow{2}{*}{$<.000$} & \multirow{2}{*}{.409} \\
\hline & Sí & 634.06 & & & \\
\hline \multirow{2}{*}{ Cu3 } & No & 759.53 & \multirow{2}{*}{200660.500} & \multirow{2}{*}{$<.000$} & \multirow{2}{*}{.414} \\
\hline & Sí & 637.90 & & & \\
\hline \multirow{2}{*}{$\mathrm{Cu} 4$} & No & 751.04 & \multirow{2}{*}{207915.000} & \multirow{2}{*}{$<.000$} & \multirow{2}{*}{.438} \\
\hline & Sí & 650.69 & & & \\
\hline \multirow{2}{*}{ Cu5 } & No & 745.70 & \multirow{2}{*}{212475.000} & \multirow{2}{*}{$<.000$} & \multirow{2}{*}{.438} \\
\hline & Sí & 658.74 & & & \\
\hline \multirow{2}{*}{ Cu6 } & No & 747.72 & \multirow{2}{*}{210747.000} & \multirow{2}{*}{$<.000$} & \multirow{2}{*}{.434} \\
\hline & Sí & 655.69 & & & \\
\hline
\end{tabular}

Finalmente, y respecto al análisis de la confianza, los resultados promedio obtenidos fueron moderados (rango medio=4). De manera más específica (tabla 11), los estudiantes se preocuparon por hacer las cosas de clase bien (rango medio=4), aprender nuevas habilidades para su proceso formativo (rango medio=4) y saber resolver problemas relacionados con su proceso formativo (rango medio=4).

Tabla 12

Dimensión confianza

\begin{tabular}{lc}
\hline Ítems & Rango medio \\
\hline Confianza_general & 4 \\
\hline Conf1 & 4 \\
\hline Conf2 & 4 \\
\hline Conf3 & 4 \\
\hline Conf4 & 4 \\
\hline Conf5 & 4 \\
\hline Conf6 & 4 \\
\hline
\end{tabular}


En esta última dimensión también se encontraron diferencias estadísticamente significativas, siendo el grupo de estudiantes con intención de abandonar los que menor valoración obtuvieron $\left(\mathrm{U}=170072.000 ; \mathrm{R}=583.95 ; \mathrm{p}<.000 ; \mathrm{PS}_{\text {est }}=.351\right)$. Así, estos estudiantes puntuaron más bajo en la realización eficiente de sus tareas académicas( $\mathrm{U}=179882.00$ $\left.0 ; \mathrm{R}=601.25 ; \mathrm{p}<.000 ; \mathrm{PS}_{\text {est }}=.371\right)$, la capacidad para hacer las cosas bien $(\mathrm{U}=198018.000 ; \mathrm{R}$ $\left.=633.24 ; \mathrm{p}<.000 ; \mathrm{PS}_{\text {est }}=.408\right)$, el aprender nuevas habilidades $(\mathrm{U}=193318.000 ; \mathrm{R}=624.95 ; \mathrm{p}<$ $\left..000 ; \mathrm{PS}_{\text {est }}=.399\right)$, trabajar de acuerdo a sus posibilidades $(\mathrm{U}=186483.500 ; \mathrm{R}=612.90 ; \mathrm{p}<.00$ $\left.0 ; \mathrm{PS}_{\text {est }}=.385\right)$, superar obstáculos $\left(\mathrm{U}=180428.000 ; \mathrm{R}=602.22 ; \mathrm{p}<.000 ; \mathrm{PS}_{\text {est }}=.372\right)$ y resolver problemas ( $\left.\mathrm{U}=178387.000 ; \mathrm{R}=598.62 ; \mathrm{p}<.000 ; \mathrm{PS}_{\text {est }}=.368\right)$.

Tabla 13

Análisis de contraste confianza

\begin{tabular}{|c|c|c|c|c|c|}
\hline Ítems & Intención de abandono & Rango promedio & $\mathrm{U}$ & $p$ & PSest \\
\hline \multirow{2}{*}{ Conf1 } & No & 783.87 & \multirow{2}{*}{179882,000} & \multirow{2}{*}{$<.000$} & \multirow{2}{*}{.371} \\
\hline & Sí & 601.25 & & & \\
\hline \multirow{2}{*}{ Conf2 } & No & 762.63 & \multirow{2}{*}{198018,000} & \multirow{2}{*}{$<.000$} & \multirow{2}{*}{.408} \\
\hline & Sí & 633.24 & & & \\
\hline \multirow{2}{*}{ Conf3 } & No & 768.13 & \multirow{2}{*}{193318,000} & \multirow{2}{*}{$<.000$} & \multirow{2}{*}{.399} \\
\hline & Sí & 624.95 & & & \\
\hline \multirow{2}{*}{ Conf4 } & No & 776.14 & \multirow{2}{*}{186483,500} & \multirow{2}{*}{$<.000$} & \multirow{2}{*}{.385} \\
\hline & Sí & 612.90 & & & \\
\hline \multirow{2}{*}{ Conf5 } & No & 783.23 & \multirow{2}{*}{180428,000} & \multirow{2}{*}{$<.000$} & \multirow{2}{*}{.372} \\
\hline & Sí & 602.22 & & & \\
\hline \multirow{2}{*}{ Conf6 } & No & 785.62 & \multirow{2}{*}{178387,000} & \multirow{2}{*}{$<.000$} & \multirow{2}{*}{.368} \\
\hline & Sí & 598.62 & & & \\
\hline
\end{tabular}

La magnitud de las diferencias encontradas en este estudio fue leve para todos los casos (Erceg-Hurn y Mirosevich, 2008).

\section{Discusión y conclusiones}

En relación al objetivo de la investigación, los resultados confirman que el dominio de competencias de adaptabilidad de carrera es un factor clave en las trayectorias formativas del alumnado universitario. Y se confirmó también la vinculación entre adaptabilidad e intención de abandono, puesto que no solo se encontró un porcentaje considerable de estudiantes que manifestaron su intención de abandonar la formación durante el periodo de confinamiento por la pandemia, sino que entre estos estaban aquellos con menos capacidad de adaptación a los cambios surgidos en el proceso de enseñanza-aprendizaje.

Se pone de manifiesto que no todas las personas tienen las mismas habilidades y recursos para afrontar los cambios y situaciones imprevistas que tienen lugar a lo largo de la vida. En el caso de los estudiantes universitarios, los cambios de escenario formativo implantados como consecuencia de la pandemia, han traído consigo dificultades que muchos no han sabido o podido gestionar. En este caso concreto, vemos que los problemas 
han sido de muy diversa naturaleza, tanto económicos, como personales y académicos. Mientras el alumnado valoró negativamente el paso a la enseñanza virtual, entre otras cuestiones por el incremento de carga lectiva, el profesorado destacó como dificultad la falta de competencias digitales básicas en los estudiantes universitarios (Tejedor et al., 2000). También Mendoza (2020) incide en la necesidad de mejorar las habilidades del alumnado para poder adaptarse a los escenarios que se han generado últimamente y que no se corresponden a la normalidad a la que estábamos acostumbrados.

La falta de apoyo de la familia, la escasez de recursos económicos, la presión que impone el confinamiento, el aislamiento, etc. han fomentado la desmotivación y con ello la tendencia a abandonar la formación. En el plano académico, el cambio acelerado a una modalidad virtual en una universidad en la que siempre se ha impartido la enseñanza de manera presencial, la falta de apoyo de los compañeros, la incapacidad de muchos docentes para hacer el tránsito al nuevo escenario formativo, el aumento de carga de trabajo, la falta de recursos, etc. han contribuido también a que el abandono académico se contemple como una escapatoria al estrés y desánimo provocado por la COVID-19. Expresiones como "estoy desmotivado", "tengo dificultades" o "no me adapto bien" reflejan claramente la falta de manejo que tiene el alumnado para hacer frente a este tipo se situaciones, que requiere, como señala Savickas (2005), capacidad para la adaptación a los cambios que se suceden de manera permanente en los distintos escenarios de la vida. Al respecto coincidimos con Figuera y Torrado (2013) cuando señalan que las posibilidades de adaptación y permanencia en los estudios pasan por una buena preparación de base, por una vinculación satisfactoria con la institución, buenas habilidades para afrontar los cambios y la intención de finalizar los estudios.

La adaptación se logra cuando las personas activan competencias adecuadas para responder a las exigencias que plantean las nuevas situaciones. En esta investigación, los estudiantes con intención de abandono fueron los que presentaron mayores problemas de adaptabilidad, ya que eran menos conscientes de las decisiones que tenían que tomar, eran menos optimistas respecto a su futuro académico profesional y no veían la necesidad de planificar sus objetivos académico-profesionales. Estos resultados coinciden con los apuntados por Romero et al. (2019), quienes encontraron también en una muestra de estudiantes universitarios debilidades importantes en factores de adaptabilidad como exploración, planificación de la carrera o control en el proceso de desarrollo vocacional. Apoyándose en Rafuse y Lanning (2016), estos autores emplearon el término "indecisión constante" para referirse a la situación que presentan muchos estudiantes que cursan estudios superiores.

En otras dimensiones de la escala de adaptabilidad se comprobó que el alumnado con intención de abandono se caracterizaba por tener menos habilidades como asumir la responsabilidad de sus acciones, confiar en sí mismos o mantenerse fiel a sus principios. Estos estudiantes que se habían planteado dejar los estudios universitarios se preocupaban menos por explorar oportunidades de crecimiento personal, investigar otras opciones antes de decidir, analizar las diferentes alternativas para lograr sus objetivos, tener menos curiosidad por encontrar otras oportunidades, etc.

Los resultados obtenidos justifican la necesidad de reforzar los sistemas de orientación y tutela del alumnado, planificando el cribado de estudiantes en riesgo de abandono para aplicar las medidas de apoyo necesario que permitan su retención y permanencia. 
La universidad actual demanda un perfil de estudiante bien orientado, con capacidad de planificar y tomar decisiones sobre su proyecto formativo y profesional, con expectativas bien definidas, con capacidad para gestionar su carrera, implicados en su proceso de aprendizaje, etc. Esto pasa por desarrollar competencias de adaptabilidad que fortalezcan este perfil de estudiantes, con verdadero sentido de pertenencia a la institución universitaria. Y el desarrollo de estas competencias no puede retrasarse hasta que el estudiante llega a la universidad. Por eso compartimos con García et al. (2016) la importancia que tiene un buen comienzo en los estudios universitarios y para ello, tal como indican Álvarez y López (2017), se debe preparar adecuadamente la transición desde las etapas previas, desarrollando competencias de adaptabilidad y creando oportunidades para la socialización académica del alumnado. A través de estas estrategias se podrán mejorar las tasas de graduación y reducir las cifras de abandono académico.

Este trabajo de investigación debe ser valorado a partir de una serie de limitaciones. En primer lugar, aunque la muestra de este trabajo cumplió con los niveles establecidos para la representatividad estadística, los resultados no pueden ser inferidos al conjunto de estudiantes de otras universidades. La preocupante situación identificada en este estudio invita a profundizar en este problema a través de diseños muestrales amplios para generalizar los resultados. Otro aspecto de interés podría ser la realización de diseños metodológicos de investigaciones longitudinales, para comprobar cómo se construye la idea de abandonar y cómo esa intención puede convertirse en una deserción definitiva. Finalmente, aunque los datos cuantitativos tienen la fortaleza de ofrecer una visión amplia de un conjunto de participantes en torno a un fenómeno objeto de estudio, en el problema del abandono académico, resulta de interés llevar a cabo investigaciones cualitativas que permitan apresar los relatos desde la perspectiva de quienes experimentan en primera persona la intención de desertar.

\section{Referencias}

Álvarez, P., y Cabrera, L. (2020). Problemas psicoeducativos del alumnado universitario y respuestas al abandono desde un enfoque orientador integral y formativo. En A. Bernardo, E. Tuero, L. Almeida y J.C. Núñez (Comp.), Motivos y factores explicativos del abandono de los estudios. Claves y estrategias para superarlo (131-142). Pirámide.

Álvarez, P., y López, D. (2011). El absentismo en la enseñanza universitaria: un obstáculo para la participación y trabajo autónomo del alumnado. Bordón, 63(3), 41-54. Recuperado de https://recyt.fecyt.es/index.php/BORDON/article/view/29054

Álvarez, P., y López, D. (2017). Recursos de Orientación para la transición académica y la toma de decisiones del alumnado: el Programa "Universitarios por un día”. Revista d'Innovació Docent Universitària, 9, 26-38. Recuperado de https://revistes. ub.edu/index.php/RIDU/article/viewFile/RIDU2017.9.3/20371

Ambiel, R.A.M., Carvalho, L., Martins, G.H., y Tofoli, L. (2016). Comparing the adaptabilities of Brazilian adolescent students and adult workers. Journal of Vocational Behavior, 94, 20-27. https://doi.org/10.1016/j.jvb.2016.02.005

Barefoot, B. (2004). Higher education's revolving door: Confronting the problem of student drop out in US colleges and universities. Open Learning, XIX(1), 9-18. https:// doi.org/10.1080/0268051042000177818 
Bernardo, A., Tuero, E., Almeida, L., y Núñez, J. (2020). Motivos y factores explicativos del abandono de los estudios. Claves y estrategias para superarlo. Pirámide.

Bethencourt, J., Cabrera, L., Hernández, J., Alvarez, P., y González, M. (2008). Variables psicológicas y educativas en el abandono universitario. Revista Electrónica de Investigación Psicoeducativa, 18, 603-622. http://dx.doi.org/10.25115/ejrep.v6i16.1298

Betz, N., y Hackett, G. (1981). The relationship of career-related self-efficacy expectations to perceived career options in college women and men. Journal of Counseling Psychology, 28(5), 399-410. https://psycnet.apa.org/doi/10.1037/0022-0167.28.5.399

Cabrera, L., Bethencourt, J., Álvarez, P., y González, M. (2006). El problema del abandono de los estudios universitarios. RELIEVE, 12(2). https://doi.org/10.7203/ relieve.12.2.4226

Erceg-Hurn. D. M., y Mirosevich. V. M. (2008). Modern robust statistical methods: an easy way to maximize the accuracy and power of your research. American Psychologist, 63(7). 591-601. https://doi.org/10.1037/0003-066X.63.7.591

Esteban, M., Bernardo, A., Tuero, E., Cerezo, R., y Núñez, J. (2016). El contexto sí importa: identificación de relaciones entre el abandono de titulación y variables contextuales. European Journal of Education and Psychology, 9, 79-88. https://doi. org/10.1016/j.ejeps.2015.06.001

Figuera, P., Torrado, M., Dorio, I., y Freixa, M. (2015). Trayectorias de persistencia y abandono de estudiantes universitarios no convencionales: implicaciones para la Orientación. Revista Electrónica Interuniversitaria de Formación del Profesorado, 18(2), 107-123. https://doi.org/10.6018/reifop.18.2.220101

Figuera, P., y Torrado, M. (2013). El contexto académico como factor diferenciador en la transición a la universidad. Revista Contrapontos, 13(1), 33-41. https://doi.org/10.14210/ contrapontos.v13n1.p33-41

Freixa, M., Llanes, J., y Venceslao, M. (2018). El abandono en el recorrido formativo del estudiante de ADE de la Universidad de Barcelona. Revista de Investigación Educativa, 36(1), 185-202. https://doi.org/10.6018/rie.36.1.278971.

García, M., Bernardo, A., y Rodríguez, L. (2016). Permanencia en la universidad: la importancia de un buen comienzo. Aula abierta, 44, 1, 1-6. https://doi.org/10.1016/j. aula.2015.04.001

García-Aretio, L. (2019). El problema del abandono en estudios a distancia. Respuestas desde el Diálogo Didáctico Mediado. Revista Iberoamericana de Educación a Distancia, 22(1), 245-270. https://doi.org/10.5944/ried.22.1.22433

García-Segura, S., Olivares-García, M., y Racionero-Siles, F. (2017). Desarrollo de la madurez vocacional en adolescentes: difícil reto para la orientación académica y profesional. Revista de Pedagogía, 38(102), 195-216. Recuperado de: https://www. redalyc.org/articulo.oa?id $=65952814010$

George, D., y Mallery, M. (2001). Using SPSS for Windows step by step: a simple guide and reference. Alyin y Bacon.

González, T., y Pedraza, I. (2017). Variables sociofamiliares asociadas al abandono de los estudios universitarios. Educatio Siglo XXI, 35(2), 365-388. https://doi. org/10.6018/j/298651

Gonzalo, F., y García, F. (2016). Permanencia y abandono de estudios en estudiantes universitarios: un análisis desde la teoría organizacional. Revista de la Educación Superior, 45(179), 25-39. https://doi.org/10.1016/j.resu.2016.06.004 
Himmel, E. (2018). Modelo de análisis de la deserción estudiantil en la educación superior. Calidad en la Educación, 17, 91-108. http://dx.doi.org/10.31619/caledu.n17.409

Holgado, F., Suárez, J. C., y Morata, M.D.L.A. (2019). Modelos de Ecuaciones Estructurales, desde el" Path Analysis" al Análisis Multigrupo: Una Guía Práctica con Lisrel. San y Torres, S.L.

Landry, C. (2003). Self-efficacy, motivation and outcome expectation correlates of college student's intention certainty. University of Southwestern Louisiana.

Mamani, T. (2017). Efecto de la adaptabilidad en el rendimiento académico. Educación Superior, 2(1), 38-44. Recuperado de: http://www.scielo.org.bo/scielo.php?script=sci_ arttext\&pid=S2518-82832017000100004\&lng=es\&tlng=es

Marín, M., Infante, E., y Troyano, Y. (2000). El fracaso académico en la Universidad: aspectos motivacionales e intereses profesionales. Revista latinoamericana de Psicología, 32(3), 505-517. Recuperado de https://hdl.handle.net/11441/73318

Martínez, L., Caraballo, A., Pérez, A., y Marcano, C. (2014). Sentido de pertenencia e inclusión social, desde las expectativas de los estudiantes de nuevo ingreso en la UDO Anaco. Saber, 26(4), 472-479. Recuperado de https://www.redalyc.org/articulo. oa? id $=427739475012$

Martínez-Clares, P., Pérez-Cusó, F.J., y Martínez-Juárez, M. (2014). Orientación Profesional en Educación Secundaria. Revista Electrónica Interuniversitaria de Formación del Profesorado, 17(1), 57-71. https://doi.org/10.6018/reifop.17.1.198841

Mendoza, L. (2020). Lo que la pandemia nos enseñó sobre la educación a distancia. Revista Latinoamericana De Estudios Educativos, 50, 343-352. https://doi. org/10.48102/rlee.2020.50.ESPECIAL.119

McMillan, J. H., y Schumacher, S. (2005). Investigación educativa: una introducción conceptual. Pearson Educación.

Muñoz, J.A., y Amón, I. (2013). Técnicas para detección de outliers multivariantes. Revista en Telecomunicaciones e Informática, 3(5), 11-25. https://revistas.upb.edu.co/ index.php/telecomunicaciones/article/view/3308

Nilforooshan, P., y Salimi, S. (2016). Career adaptability as a mediator between personality and career engagement. Journal of Vocational Behavior, 94, 1-10. https://doi. org/10.1016/j.jvb.2016.02.010

Oviedo. H. C., y Campo-Arias. A. (2005). Aproximación al uso del coeficiente alfa de Cronbach. Revista Colombiana de Psiquiatría, 34(4), 572-580. https://bit.ly/330x2Ip

Perera, H.N., y Mcllveen, P. (2017). Profiles of career adaptativity and their relations with adaptability, adapting and adaptation. Journal of Vocational Behavior, 98, 70-84. https://doi.org/10.1016/j.jvb.2016.10.001

Pérez-López, E., Vázquez, A., y Cambero, S. (2021). Educación a distancia en tiempos de COVID-19: Análisis desde la perspectiva de los estudiantes universitarios. RIED. Revista Iberoamericana de Educación a Distancia, 24(1), http://dx.doi.org/10.5944/ ried.24.1.27855Rafuse, A., y Lanning, R. (2016). Knowledge and risk: choices and decisions in the career selection process among University students. The Canadian Journal of Career Devolpment,15(2), 32-42. Recuperado de http://cjcdonline. $\mathrm{ca} /$ download/knowledge-risk-choices-decisions-career-selection-process-amonguniversity-students/ 
Rausch, J., y Hamilton, M. (2006). Goals and Distractions: Explanations of Early Attrition from Traditional University Freshmen. The Qualitative Report, 11(2), 317-334. https:// doi.org/10.46743/2160-3715/2006.1676

Raykov, T., y Marcoulides, G.A. (2017). Thanks Coefficient Alpha, We still need you! Educational and Psychological Measurement, 79(1), 200-210. https://doi. org/10.1177/0013164417725127

Romero, S., y Figuera, P. (2016). Orientación en la Universidad. En A. Manzanares-Moya y C. Sanz-López (Dir.), Orientación Profesional. Fundamentos y estrategias (163-181). Walters Kluwer.

Romero, S., Figuera, P., Freixa, M. y Llanes, J. (2019). Adaptabilidad de la carrera en estudiantes universitarios: un estudio a través de entrevistas autobiográficas. Revista de Investigación Educativa, 37(2), 379-394. http://dx.doi.org/10.6018/rie.37.2.322441

Salavera, C., y Usán, P. (2017). Repercusión de las estrategias de afrontamiento de estrés en la felicidad de los alumnos de Secundaria. Revista electrónica interuniversitaria de formación del profesorado, 20(3), 65-77. http://dx.doi.org/10.6018/reifop.20.3.282601

Santana, L., Medina, P., y Feliciano, L. (2019). Proyecto de vida y toma de decisiones del alumnado de Formación Profesional. Revista Complutense de Educación, 30(2), 423-440. https://doi.org/10.5209/RCED.57589

Savickas, M.L. (2005). The theory and practice of career construction. En S.D. Brown y R.W. Lent (eds.), Career Development and Counseling: Putting theory and research to work (42-70). John Wiley \& Sons.

Savickas, M.L., y Porfeli, E.J. (2012). Career Adapt-Abilities Scale: Construction, reliability, and measurement equivalence across 13 countries. Journal of Vocational Behavior, 80, 661-673. https://doi.org/10.1016/j.jvb.2012.01.011

Tejedor, S., Cervi, L., Tusa, F., y Parola, A. (2020). Educación en tiempos de pandemia: reflexiones de alumnos y profesores sobre la enseñanza virtual universitaria en España, Italia y Ecuador. Revista Latina de Comunicación Social, 78, 1-21. https://www. doi.org/10.4185/RLCS2020-1466Tuero, E., Cervero, A., Esteban, M., y Bernardo, A. (2018). ¿Por qué abandonan los alumnos universitarios? variables de influencia en el planteamiento y consolidación del abandono. Educación XX1, 21 (2), 174-5374. https://doi.org/10.5944/educxx1.20066

Viladrich, C., Angulo-Brunet, A., y Doval, E. (2017). Un viaje alrededor de alfa y omega para estimar la fiabilidad de consistencia interna. Anales de Psicología, 33(3), 755-782. http://dx.doi.org/10.6018/analesps.33.3.268401

Wilkins, K., Roach, Ch., Tracey, T. y Yel, N. (2018). The effects of career adaptability on intended academic persistence: The mediating role of academic satisfaction. Journal of Vocational Behavior, 108, 67-77. https://doi.org/10.1016/j.jvb.2018.06.006.

Zepke, N., y Leach, L. (2010). Improving Student Engagement: Ten Proposals for Action. Active Learning in Higher Education, lol(3), 167-177. https://doi. org/10.1177/1469787410379680

Fecha de recepción: 16 de enero de 2021.

Fecha de revisión: 10 de febrero de 2021.

Fecha de aceptación: 10 de junio de 2021. 